\title{
Inovação e sistemas de inovação: relevância para a área de saúde
}

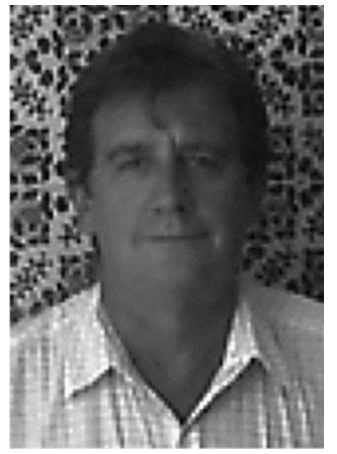

José Eduardo

Cassiolato

RedeSist (Rede de Pesquisa em Sistemas Produtivos e Inovativos Locais): Instituto de Economia - Universidade Federal do Rio de Janeiro (IE/UFRJ), Rio de Janeiro, Brasil cassio@ie.ufrj.br

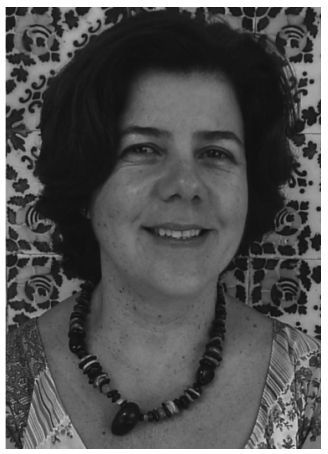

Helena M.

M. Lastres

RedeSist (Rede de Pesquisa em Sistemas Produtivos e Inovativos Locais): Instituto de Economia - Universidade Federal do Rio de Janeiro (IE/UFRJ), Rio de Janeiro, Brasil hlastres@ie.ufrj.br

\section{Resumo}

Nas últimas décadas do século XX, atenção especial passa a ser dada aos processos de geração, difusão e uso de conhecimentos. Diversos enfoques analíticos e normativos têm sido desenvolvidos visando entender e orientar esses processos. Este artigo objetiva apresentar e discutir os conceitos e sistema de inovação, suas vantagens e desafios, assim como examinar a experiência brasileira na sua utilização e no seu desenvolvimento. Em seu final, o artigo retoma as principais conclusões da análise realizada, destacando: (i) a prioridade de estimular e desenvolver os sistemas produtivos e inovadores mobilizadores do desenvolvimento social - como os de saúde; (ii) a urgência em avançar na compreensão das possibilidades de desenvolvimento dessas áreas, assim como na formulação de políticas que orientem e dinamizem esse desenvolvimento de forma sistêmica e sustentável.

\section{Palavras-chave}

Inovação, sistemas de inovação, arranjos e sistemas produtivos locais, experiência brasileira, áreas mobilizadoras do desenvolvimento social

\section{Introdução}

A atenção dada às formas de geração, difusão e uso de conhecimentos ganhou renovado vigor nas últimas décadas do século XX. Dentre os principais avanços, destaca-se o desenvolvimento do conceito de sistemas de inovação crescentemente utilizado para entender o papel da inovação e do conhecimento na competitividade de organizações e países. O foco deixa de ser as inovações e organizações individuais, passando a se concentrar nos processos sistêmicos que permitem às empresas e demais organizações aprender, usar e acumular capacitações e desenvolver novos produtos e processos (FREEMAN, 1982a; 1987; LUNDVALL, 1985; IMAI et al., 1989). Retomar esta discussão é um dos objetivos deste texto.
Um segundo objetivo é o de discutir a experiência brasileira na utilização no desenvolvimento desse conceito, tanto na análise, quanto na orientação das capacitações produtivas e inovativas. Já o terceiro objetivo é o de alertar para a urgência de estimular e desenvolver os sistemas produtivos e inovativos mobilizadores do desenvolvimento social no Brasil - como os de saúde

$\mathrm{O}$ artigo encontra-se estruturado da seguinte forma: o item 2 discute os desenvolvimentos do conceito de inovação desde o final dos anos 1960s, culminando com a evolução do conceito de sistemas de inovação, nos anos 1980s. O item 3 apresenta a evolução deste conceito, discutindo suas vantagens e desafios e apontando alguns do principais pontos de conexão entre as contribuições 
entre a chamada Escola Estruturalista Cepalina e a Escola Neo-schumpeteriana. O item 4 examina a experiência brasileira na utilização no desenvolvimento do conceito de sistemas produtivos e inovativos locais. Na conclusão, aponta-se a necessidade de avançar tanto no entendimento dos sistemas produtivos e inovativos mobilizadores do desenvolvimento social, como na formulação de políticas que orientem e dinamizem o desenvolvimento dos mesmos de forma sistêmica e sustentável.

\section{Inovação}

A literatura sobre inovação tem em sua fundação as contribuições de Schumpeter, e em especial sua tentativa de teorização da relação entre inovação tecnológica e o desenvolvimento econômico. O crescimento da economia é visto como um processo dinâmico que depende tanto da geração e uso das inovações, quanto dos processos de difusão das mesmas. Os avanços - produtivos, tecnológicos, organizacionais, institucionais, etc. - resultantes de processos inovativos são tomados com fator básico na formação dos padrões de transformação da economia e de seu desenvolvimento no longo prazo (SCHUMPETER, 1912; 1939; 1942). Essas contribuições têm sido qualificadas e aprimoradas por uma série de autores que o seguiram na busca de entender a dinâmica capitalista focalizando centralmente a dinâmica inovativa e seus impactos no desenvolvimento de organizações e países.

Até os anos 1960, a inovação era identificada com novos produtos ou processos e entendida como ocorrendo em estágios sucessivos de pesquisa básica, pesquisa aplicada, desenvolvimento, produção e difusão (visão linear da inovação). Geralmente a discussão sobre as fontes mais importantes de inovação, polarizava-se entre aqueles que (i) atribuíam maior importância ao avanço do desenvolvimento científico (science push) e os que (ii) destacavam a relevância das pressões da demanda por novas tecnologias (demand pull).

Nas décadas seguintes, o entendimento da inovação é requalificado e ampliado, com amplas conseqüências para a esfera das políticas de ciência e tecnologia (C\&T). Estudos empíricos e teórico-conceituais mostraram que existe uma ampla gama de informações e conhecimentos essenciais favorecendo a geração e incorporação de novidades (inovação), processos estes caracterizados por mecanismos de tentativa e erro e de feedbacks. As inovações passaram a ser entendidas como resultantes do conjunto de atividades interligadas, compreendendo principalmente sua assimilação, uso e difusão. A análise do processo inovativo passa a se concentrar nas estruturas subjacentes a tais conexões. Reconheceu-se, por exemplo, que apesar de o processo de acumulação de conhecimentos ser essencialmente específico da empresa ele é fundamentalmente influenciado por constantes relações entre firmas e demais organizações. Portanto, a inovação passa a ser entendida não como "um único ato, mas sim uma série deles ... adquirindo significado econômico apenas através de extenso processos de redesign, modificação e inúmeras pequenas melhorias" (ROSENBERG, 1976) Ou como preferiu DOSI (1988), "a busca por e a descoberta, experimentação, desenvolvimento, imitação e adoção de novos produtos, novos processos produtivos e novos sistemas organizacionais".

Especialmente importante foi o entendimento de que os processos de inovação e de difusão se determinam mútua e simultaneamente. Constatou-se na verdade que ao se iniciar o processo de difusão de qualquer tecnologia existe um conjunto de novidades concorrentes, baseadas em tecnologias que se alteram constante e sistematicamente em resposta à experiência e aos incentivos que surgem durante a difusão (METCALFE, 1986). Nesta perspectiva, interações entre produtores e usuários e a existência de um pool relativamente sofisticado de qualificações no entorno são elementos importantes no processo de desenvolvimento de uma nova tecnologia. Processos de seleção sociais, econômicos e políticos vinculados à geração, uso de difusão de inovações contribuem simultaneamente para definir as trajetórias tecnológicas.

Trata-se, portanto, de uma relação biunívoca, onde o ambiente no qual a inovação se desenvolve e difunde conforma o padrão da evolução das tecnologias, que por sua vez redefine a própria trajetória inovativa. Ambientes diferentes onde se encontram as empresas e organizações são associados a diversos padrões de avanço tecnológico (GEORGHIOU et al., 1986). Isto ressalta as especificidades nacionais, regionais e locais dos processos de geração, uso e difusão de inovações. O processo inovativo é então visto como resultado da aprendizagem coletiva, a partir dos vínculos dentro da empresa e entre esta e demais organizações (LUNDVALL, 1985; PÉREZ, 1988). A inovação passou a ser vista, não mais como um ato isolado, mas sim como um processo de aprendizado interativo, não-linear, cumulativo, específico da localidade e dificilmente replicável.

O caráter sistêmico da inovação já era reconhecido por FREEMAN (1982a), que apontava que as decisões e estratégias tecnológicas são dependentes de fatores que abrangiam o setor financeiro, o sistema de educação e a organização do trabalho, além da própria esfera da produção e comercialização de bens e serviços. Este esforço antecipou a definição do conceito de sistema nacional de inovação, a qual foi explicitada no livro sobre a evolução do caso japonês (FREEMAN, 1987). É interessante notar que diversos autores latino-americanos e caribenhos, pelo menos desde meados do século XX, sempre apontaram que o entendimento da dinâmica industrial e tecnológica, e das políticas para sua mobilização, exige considerar e atuar de forma sistêmica os condicionantes do quadro macroeconômico, político, institucional e financeiro específico de cada país (PREBISCH, 1949; FURTADO, 1961). Uma percepção fundamental de que este contexto mais amplo jamais pode ser ignorado foi a observação de que o mesmo se constitui em importante "política implícita" capaz de dificultar e até anular as políticas explícitas específicas (HERRERA, 1971).

Significativo, também, é que os trabalhos de FREEMAN (1982a; b), que associando o entendimento da evolução do capitalismo às ondas de crescimento e depressão de longo prazo: 
- exploram as formas do processo inovativo no novo paradigma tecnológico das TICs;

- criticam as teses de que o livre comércio seria desvantajoso para países menos desenvolvidos;

- apontam a necessidade de iniciativas governamentais para dar conta da incertezas especialmente altas nos períodos de mudança de paradigma.

As mudanças nos paradigmas técnico-econômicos são tidas como essenciais para explicar os períodos de crescimento e de crise econômica. Os novos paradigmas alteram as fronteiras tecnológicas e criam novos conjuntos de padrões, práticas e processos produtivos. Geralmente a mudança tecnológica é rápida e acompanhada por um grande nível de inércia nas instituições e organizações públicas e privadas. Deste modo, os períodos de crise são vistos a partir do conflito entre a emergência do novo paradigma e a estrutura institucional anterior, assim como os booms econômicos são relacionados aos períodos em que ocorre a adaptação das instituições e da estrutura econômica e a sua interação com o novo paradigma tecno-econômico (FREEMAN, 1982c; 1998; PEREZ, 1983; 1988).

Estes desenvolvimentos levaram à ênfase da visão sistêmica nas proposições de política e à relevância de focalizar as articulações entre os diferentes atores nos distintos sistemas nacionais de inovação. Destaca-se a dupla característica das novas políticas: a inovação passa a ser o mais importante componente das estratégias de desenvolvimento (e não apenas das políticas de C\&T ou das políticas industriais) e as políticas a ela direcionadas passam a ser entendidas como políticas para sistemas de inovação.

O foco em conhecimento, aprendizado e interatividade deu sustentação à idéia de sistemas de inovação, os quais foram conceituados como conjuntos de instituições que contribuem para e afetam o desenvolvimento da capacidade de aprendizado, criação e uso de competências de um país, região, setor ou localidade (FREEMAN, 1987; 1988; LUNDVALL, 1992; 1995). Tais sistemas constituem-se de elementos que interagem na produção, uso e difusão do conhecimento. Estes sistemas contêm, não apenas aqueles diretamente voltadas ao desenvolvimento científico e tecnológico, mas vários outros, incluindo formas de comportamento, normas, políticas e demais características do contexto onde se localizam. Reforça-se, deste modo, a idéia que os processos de inovação - que têm lugar no nível da firma - são também gerados e sustentados por suas relações com outras empresas e organizações e dependem deste ambiente mais amplo.

\section{A evolução do conceito de sistemas de inovação, suas vantagens e desafios}

Conforme apontado por LUNDVALL (2006), alguns autores tendem a utilizar o conceito de sistemas de inovação de forma restrita, considerando-o como um desdobramento de visões anteriores sobre os sistemas nacionais de ciência e tecnologia (NELSON, 1993; MOWERY et al., 1995). Para esta linha de raciocínio, os pontos principais se relacionam a mapear indicado- res de especialização e desempenho nacionais relativos aos esforços de P\&D e inovação e às organizações de C\&T. No que se refere à política, os tópicos se referem exclusivamente à política explícita de C\&T. A análise inclui alguns fatores que influenciam a produção e uso do conhecimento, mas ignora o conjunto mais amplo de elementos: desde aqueles que conformam a criação de capacitações - tais como a educação, treinamento, relações industriais e a dinâmica do mercado de trabalho - até aqueles outros mais gerais mas que afetam decisivamente os sistemas de inovação, como as políticas implícitas macro-econômicas e o setor financeiro.

A definição mais ampla de sistemas nacionais de inovação inclui estas dimensões analíticas, incorporando o papel das firmas, organizações de ensino e pesquisa, governo (como um todo e não apenas a política de $C \& T$ ), organismos de financiamento, e outros atores e elementos que influenciam a aquisição, uso e difusão das inovações. Nesta linha é que se enfatiza (i) o papel de processos históricos - responsáveis por diferenças em trajetórias de desenvolvimento, evolução político-institucional e capacitações sócio-econômicas; (ii) a importância do caráter nacional dos sistemas de inovação. (FREEMAN, 1982; 1987; LUNDVALL, 1985). Conforme destacado acima, desde o primeiro trabalho que introduziu o conceito, FREEMAN (1982a), argumentava que não apenas o desempenho dos países está ligado à inovação, mas que outros fatores além das organizações de C\&T e P\&D influenciam significativamente o desempenho inovativo de países e empresas, sublinhando a sua natureza nacional. Posteriormente ele usou especificamente o conceito amplo de sistemas nacionais de inovação na análise do desempenho econômico e tecnológico japonês dos anos 1950s até os 1980s.

A abordagem de sistemas nacionais de inovação foi também ampliada por trabalhos que destacaram a relevância das relações produtor-usuário para a inovação e o papel do mercado doméstico (LUNDVALL, 1988). Tal trabalho destacou que uma importante fonte de inovação é o aprendizado interativo que ocorre na produção, desenvolvimento tecnológico, marketing, vendas e que envolve elementos não vinculados ao preço tais como poder, lealdade e confiança. Tudo isto reafirmou a importância de capturar a especificidade dos diferentes atores, o tipo e a qualidade das relações e o entendimento do papel das instituições no seu sentido mais amplo - como normas e regras, informais e formais. Ênfase especial foi dada a este papel das instituições na determinação: (i) de como as pessoas se relacionam e como elas aprendem e usam conhecimentos; (ii) da direção que tomam e da taxa em que evoluem as atividades inovativas (JOHNSON, 1992; LUNDVALL, 2006; JOHNSON et al., 2003).

Evidentemente que continuam tendo enorme utilidade as análises focalizando as relações produtorusuário, universidade-empresa, etc. No entanto, não se deve esquecer todo o avanço registrado nas últimas três décadas no entendimento da inovação - como processo sistêmico, com múltiplas e simultâneas fontes e não-linear - o qual nos faz compreender que tais relações em alguns sistemas esta relações podem até ser as principais, 
mas jamais serão as únicas. Adicionalmente, destaca-se que em todos os países é reconhecida a importância de processos formais e informais de geração, aquisição, uso e disseminação de conhecimentos. No caso das relações universidade-empresa destaca-se, portanto, a restrição a este particular tipo de organização de ensino e pesquisa. ${ }^{1}$ Um enfoque mais amplo deve, não apenas considerar o contexto das organizações de ensino e pesquisa com um todo (incluindo universidades, escolas e centros de capacitação de diversos níveis), mas também ser capaz de apreender os processos informais envolvidos nos processos de aprendizagem e criação de capacitações.

Reitera-se aqui a conclusão que para explicar o desempenho econômico é necessário considerar as dimensões sociais, políticas e culturais específicas a cada realidade. Isto por sua vez reforça a necessidade de um instrumental analítico/normativo mais amplo e complexo do que aquele oferecido pela teoria econômica tradicional. Nesta direção, tanto FREEMAN quanto LUNDVALL assinalam as restrições da análise quantitativa baseada em modelos abstratos, propondo um método por eles caracterizado como 'reasoned history'. Citando SCHUMPETER na análise do desenvolvimento econômico eles sublinham que (FREEMAN 1982a; LUNDVALL, 2006):

\begin{abstract}
“... it is absurd to think that we can derive the contour lines of our phenomena from our statistical material only. All we could ever prove from it is that no regular contour lines exist ... We cannot stress this point sufficiently. General history (social, political and cultural), economic history and more particularly industrial history are not only indispensable, but really the most important contributors to the understanding of our problem. All other materials and methods, statistical and theoretical, are only subservient to them and worse than useless without them."
\end{abstract}

Outra dimensão que constitui uma parte importante do entendimento mais amplo dos sistemas nacionais de inovação - e que tem um impacto significativo na inovação - é a perspectiva de tempo. Como exemplo de como diferenças institucionais têm uma influência decisiva na conduta e desempenho nacionais, as estratégias corporativas de curto prazo têm sido confrontadas com perspectivas de longo prazo. Tal fator é ainda mais relevante no caso de investimentos em atividades que necessitam maturação no longo prazo e envolvem alto risco, como é o caso de educação e inovação. Outra dimensão se refere ao papel da confiança e das instituições a ela relacionadas. A força e o tipo da confiança determinarão como o aprendizado interativo ocorrerá. Arranjos formais e legais refletirão e terão um impacto nesta dimensão social tácita. Outras instituições formais e informais que são importantes para o sistema nacional de inovação incluídas na definição mais ampla são: o nível de coesão e solidariedade, o papel da educação e treinamento, mercado de trabalho e legislação corporativa, legislação contratual, instituições de arbitragem, etc. Todas são historicamente determinadas e dependentes do contexto (JOHNSON et al., 2003; LUNDVALL, 2006).

Foi particularmente relevante o fato de o conceito de sistema de inovação haver sido criado e desenvol- vido em meados dos anos 1980, exatamente quando tomava corpo e rapidamente se difundia a tese sobre a aceleração da globalização econômica, a qual inclusive foi associada à hipótese de uma tendência a um suposto tecnoglobalismo ${ }^{2}$.

Como vimos, esta abordagem reforçou o foco no caráter cumulativo localizado e nacional da geração, assimilação e difusão da inovação, assim como a conclusão que a base do dinamismo e da competitividade das empresas não se restringe:

- a uma única empresa ou a um único setor, estando fortemente associada a atividades e capacidades existentes ao longo da cadeia de produção e comercialização, além de envolver uma série de atividades e organizações responsáveis pela assimilação, uso e disseminação de conhecimentos e capacitações;

- apenas aos atores econômicos e às cadeias e complexos produtivos, mas reflete também as particularidades dos demais atores sociais e políticos, assim como dos ambientes onde se inserem.

Assim, diferentes contextos, sistemas cognitivos e regulatórios e modos de articulação e de aprendizado são reconhecidos como fundamentais na aquisição, uso e difusão de conhecimentos e particularmente aqueles tácitos. Tais sistemas e modos de articulação podem ser tanto formais como informais.

Outro avanço crucial consolidado na abordagem de SIN se refere à constatação de que inovação não se restringe a processos de mudanças radicais na fronteira tecnológica, realizados quase que exclusivamente por grandes empresas através de seus esforços de pesquisa e o desenvolvimento (P\&D). São significativas as conseqüências de entender a inovação como "processo pelo qual as organizações incorporam conhecimentos na produção de bens e serviços que lhes são novos, independentemente de serem novos, ou não, para os seus competidores domésticos ou estrangeiros". ${ }^{3}$ Esse entendimento ajuda a evitar diversas distorções, incentivando os policy-makers a adotarem uma perspectiva mais ampla sobre as oportunidades para o aprendizado e a inovação em pequenas e médias empresas (PMEs) e também nas chamadas indústrias tradicionais. As implicações para políticas de tais qualificações são significativas.

Assim, em vez de ignorar as especificidades dos diferentes contextos e atores locais, os principais blocos do enfoque em sistemas de inovação exigem que sejam elas sejam captadas e analisadas. A ênfase em tratar a inovação como um processo cumulativo e específico ao contexto determinado permite desmistificar, por exemplo, idéias simplistas sobre as possibilidades de gerar, adquirir e difundir tecnologias. Tal ênfase torna claro que a aquisição de tecnologia no exterior não substitui os esforços locais. Ao contrário, é necessário muito conhecimento para poder interpretar a informação; selecionar, comprar, copiar, transformar e internalizar a tecnologia importada.

Outro aspecto essencial é o papel central dado à inovação para a competitividade dinâmica e sustentável. Esta contrasta com a usual prioridade dada à exploração 
das vantagens competitivas tradicionais (como baixos custos da mão-de-obra e da exploração de recursos naturais sem uma perspectiva de longo prazo e à manipulação da taxa de câmbio), as quais FAJNZYLBER (1988) chamou de espúrias.

Apesar destas e outras vantagens associadas ao desenvolvimento e uso da abordagem em sistemas de inovação, alertas têm sido feitos sobre o risco de estes representarem apenas rótulos novos em velhas práticas. Refere-se aqui àquele enunciado por REINERT et al. (2003), que algumas tentativas de uso do enfoque em sistemas de inovação não passavam de 'a thin icing on a solid neo-classical cake' :

'We argue that by integrating some Schumpeterian variable to mainstream economics we may not arrive at the root causes of development. We risk applying a thin Schumpeterian icing on what is essentially a profoundly neoclassical way of thinking, trade theory is but one example here. ... As has already frequently been emphasized in the NIS approach, it is crucial to understand the different national contexts.' (REINERT et al, 2003).

A tentativa de dar um caráter operacional ao conceito de sistemas de inovação tem levado diversos autores a propor diferentes dimensões a ele associadas. Assim a idéia de sistemas supranacionais (FREEMAN, 1999), regionais (COOKE et al., 1998) e setoriais de inovação (MALERBA et al., 1996) tem sido proposta pela literatura. Em paralelo, a percepção sobre a importância da proximidade geográfica das empresas para explicar um bom desempenho na competitividade de firmas tem aumentado. Termos como sinergia, economias de aglomeração (clustering), eficiência coletiva, etc têm sido apresentados de modo a exprimir algumas preocupações de tal debate. A pesquisa sobre aglomerações industriais e sobre o local como uma fonte de vantagens competitivas tem crescido significativamente nos últimos anos. A idéia de aglomerações setoriais torna-se associada ao conceito de competitividade a partir do início dos anos 1990 e tem sido utilizada, tanto como unidade de análise, quanto como unidade de ação de políticas industriais.

Evidentemente existem diferenças entre uma visão estritamente setorial da inovação e a abordagem sistêmica apresentada neste artigo. De fato, a visão setorial não captura a situação atual onde as fronteiras dos setores produtivos encontram-se em mutação, tornando-se fluidas. Questionam-se também as formas tradicionais de mensurar e avaliar atividades econômicas agrupando-as em setores, principalmente dada a heterogeneidade das organizações e suas estruturas produtivas e inovativas existentes dentro de um mesmo setor. Adiciona-se a esta condição a tendência tanto à incorporação de conhecimentos avançados e crescentemente multidisciplinares, como à convergência das funções e aparatos tecnológicos de vários segmentos até então desvinculados entre si. Tais tendências são particularmente marcante em situações de transformações técnico-econômicas radicais e abrangentes - como nas mudanças de paradigma (LASTRES et al., 2006). Há casos ilustrativos nos chamados setores primários, como o agrícola, o extrativo e o pesqueiro, e também naqueles mais avançados.
MARQUES (1999), por exemplo, utiliza a produção de tomates para mostrar como as novas tecnologias afetam todas as etapas da cadeia produtiva, sugerindo que a produção deste bem depende e se articula profundamente com a produção de diversos setores, tornando pouco relevante a sua classificação como um produto agrícola: "agora, antes de plantar tomates são necessários muitos planos, desenhos, tabelas e roteiros para produzir as sementes geneticamente tratadas, os fertilizantes, o plantio geométrico, a colheitadeira, o sistema de seleção eletrônica, os recipientes e seus meios de transporte, etc. ... - o tomate é um produto high-tech!"

Aponta-se que com a alta difusão das novas tecnologias base do novo padrão - TICs, biotecnologia, engenharia genética e materiais avançados - mesmo setores considerados tradicionais podem apresentar-se como intensivos em tecnologias de ponta. Com isto se torna ainda mais evidente a inadequação da forma como são definidos os setores econômicos. Apesar de o conhecimento já acumulado sobre as trajetórias setoriais continuarem relevantes, tanto a produção quanto a inovação são cada vez mais influenciadas pelo conhecimento e as capacidades de diferentes atividades produtivas e áreas científicas e tecnológicas. Devido às dificuldades em mensurar os conhecimentos de variadas origens utilizados nos diferentes setores, continuamos tratando tais setores do mesmo modo que quando as classificações foram concebidas. Portanto, mesmo que sejam adicionadas novas atividades e setores àqueles que fazem parte dos sistemas estatísticos dos diferentes países, mostra-se crescentemente difícil continuar usando estas categorias sem questioná-las (LASTRES et al., 2006).

As principais conclusões desta discussão contribuem para ressaltar a necessidade de um referencial que dê conta dos novos desafios. A classificação setorial usual relaciona-se a conjuntos de conhecimentos e atividades que podem agora estar representando peso minoritário no valor agregado do setor em questão. Evidentemente a linha de fronteira entre setores sempre foi arbitrária. Ressaltamos porém que, no quadro atual, torna-se mais agudo o problema de se captar - através de indicadores imperfeitos - apenas parte dos sistemas produtivos e inovativos.

Existe, portanto, uma necessidade de avançar no refinamento do uso da visão sistêmica, tanto no âmbito analítico quanto político-normativo. O objetivo do próximo item é apresentar brevemente a experiência brasileira no desenvolvimento e uso pragmático do conceito de sistema nacional de inovação.

\section{A experiência brasileira no desenvolvimento do conceito de sistema de inovação}

No Brasil, o conceito de sistemas produtivos e inovativos locais foi criado e desenvolvido pela RedeSist em finais da década de 1990s e foi rapidamente disseminado na esfera de ensino e pesquisa e de política (CASSIOLATO et al., 1999; 2005; LASTRES et al., 1999; 2006). Este conceito combina as contribuições sobre desenvolvimento da escola estruturalista latino 
americana com a visão neo-schumpeteriana de sistemas de inovação. ${ }^{4}$ Chama-se a atenção para o significativo processo de aprendizado posto em marcha ao colocar em prática esta nova abordagem, tanto enquanto ferramenta analítica quanto de orientação de políticas. Todos os atores envolvidos aprenderam muito com erros e acertos e muitas vezes tiveram que inovar.

De acordo com a definição proposta pela RedeSist ${ }^{5}$, sistemas produtivos e inovativos locais (SPILs) designa conjuntos de atores econômicos, políticos e sociais, localizados em um mesmo território, que apresentam vínculos ao desempenharem atividades de produção e inovação. SPILs geralmente incluem:

- empresas - produtoras de bens e serviços finais; fornecedoras de bens e serviços (matérias-primas, equipamentos e outros insumos); distribuidoras e comercializadoras; consumidoras, etc. - atuando tanto nos setores primário e secundário quanto no terciário;

- organizações voltadas à formação e treinamento de recursos humanos, informação, pesquisa, desenvolvimento e engenharia, promoção e financiamento, etc..

- cooperativas, associações, sindicatos e demais órgãos de representação;

Arranjos produtivos locais APLs designa os casos de sistemas fragmentados e desarticulados.

Seguindo as orientações do foco em sistemas de inovação, esta abordagem focaliza conjuntos de diferentes atores, assim como atividades conexas dos diferentes sistemas produtivos e inovativos locais. Acompanhando o desenvolvimento deste conceito, a RedeSist também desenvolveu uma metodologia que focaliza e investiga as articulações entre empresas e destas com outros atores; os fluxos de conhecimento (em particular, em sua dimensão tácita); as bases dos processos de aprendizado para capacitação produtiva, organizacional e inovativa; e o papel da proximidade geográfica e da identidade histórica, institucional, social e cultural como fontes de diversidade e vantagens competitivas sustentadas. $\mathrm{O}$ objetivo final é discutir as implicações para políticas das análises realizadas ${ }^{6}$.

As experiências pioneiras de analisar e promover sistemas produtivos e inovativos no Brasil confirmam que esta consiste de fato em uma nova forma de pensar e fazer política que:

- coloca a geração, aquisição e difusão de conhecimentos e a criação e uso de capacitações produtivas e inovativas como fatores chave da produtividade e competitividade dinâmica e duradoura de organizações, regiões e países;

- engloba diferentes tipos de atores e atividades, inclusive aqueles geralmente excluídos das ações de promoção, com por exemplo as empresas de micro e pequeno portes e seus requerimentos; as atividades do setor primário e terciário, os segmentos à margem da vida econômica formal - empresas, atividades e processos de aquisição, transmissão de conhecimento;

- cobre o espaço, onde ocorre o aprendizado, são criadas as capacitações produtivas e inovativas e fluem os conhecimentos e particularmente aqueles tácitos;
- permite estabelecer uma ponte entre o território e as atividades econômicas, as quais também não se restringem aos cortes clássicos espaciais como os níveis municipais e de micro-região;

- visa dar conta das variações espaciais devidas à grande extensão geográfica, heterogeneidade e desigualdades econômicas, políticas, sociais e regionais;

- tem proporcionado um entendimento amplo das oportunidades e desafios colocadas ao desenvolvimento produtivo e inovativo;

- representa o nível em que as políticas de promoção do aprendizado e criação de capacitações produtivas e inovativas podem ser mais efetivas;

- destaca a necessidade de articular e implementar as diferentes políticas numa perspectiva integrada e de longo prazo.

Segundo esse enfoque, onde houver produção de qualquer bem ou serviço haverá sempre um sistema em torno da mesma, envolvendo atividades e atores relacionados desde a aquisição de matérias-primas, máquinas e demais insumos até a sua comercialização. Tais sistemas variarão desde aqueles mais rudimentares àqueles mais complexos e articulados, que funcionam de modo realmente sistêmico. Nesta perspectiva, o número de sistemas produtivos locais existentes em qualquer país é tão grande quanto sua capacidade produtiva permita. Tanto do ponto de vista analítico quanto normativo, não basta desenvolver indicadores e mapas objetivando identificar a quantidade de sistemas existentes e suas diferentes configurações e graus de desenvolvimento. De forma semelhante, por serem baseadas no reconhecimento das especificidades dos diferentes sistemas, as políticas para sua promoção são incompatíveis com modelos genéricos que utilizam idéias de benchmark e best practice.

Diferentes tipologias e indicadores vêm sendo desenvolvidos visando entender os processos de aprendizado, capacitação e inovação. Entretanto, alerta-se que o uso de algumas dessas taxonomias, indicadores, assim como a seleção de casos exemplares não deve de maneira alguma inibir a compreensão dos elementos diferenciados que a riqueza das experiências apresenta no mundo real. Isto é particularmente importante no caso da definição e implementação de políticas. Sublinha-se aqui a conclusão que a adoção de políticas uniformes ignora a existência de disparidades, que decorrem não só de fatores econômicos, mas também de diversidades das matrizes sócio-políticas e das particularidades históricas (FURTADO, 1998). A mobilização de um determinado sistema produtivo geralmente implica em conjuntos específicos de requerimentos que variam tanto no espaço e quanto no tempo.

\section{Sugestões de política para mobilização de arranjos e sistemas produtivos e inovativos no Brasil}

A promoção de sistemas produtivos e inovativos tem sido vista como uma nova forma de política para o desenvolvimento industrial e tecnológico capaz de dar 
conta das especificidades do novo padrão de acumulação. No entanto, para que a ênfase em APLs corresponda não apenas meramente a utilização de novos rótulos em velhas práticas de forma a seguir a 'moda' e para se obter acesso a apoio financeiro, as abordagens analíticas e normativas devem avançar e incorporar de fato a essência dos conceitos envolvidos. Aponta-se, assim, para a necessidade de uma melhor compreensão dos conceitos de desenvolvimento, competitividade, inovação e APLs entre os pesquisadores, formuladores e executores de políticas, tanto públicas quanto privadas.

Particularmente urgente é avançar no entendimento e uso coerente do conceito, assim como superar suposições que colocam (i) a promoção da inovação e da competitividade como oposta à promoção do desenvolvimento local e da inclusão social; (ii) o desenvolvimento local como sinônimo de fragmentação do espaço nacional. Adicionalmente, coloca-se a necessidade de formular e implementar políticas adaptadas aos desafios brasileiros e que promovam um desenvolvimento mais amplo e inclusivo, em vez de apostar sempre e exclusivamente nos mesmos "vencedores", o que contribui para reforçar as desigualdades regionais e sociais.

Nesta linha, recomenda-se que as discussões sobre política para promoção de APLs iniciem com a superação da visão enviesada e parcial sobre os processos de desenvolvimento e dos modelos genéricos de política - os quais têm como referência o desempenho das maiores e mais dinâmicas empresas do mundo e as instituições de alguns países desenvolvidos - e com o necessário desenvolvimento de referenciais conceituais próprios e capazes tanto de captar as especificidades do desenvolvimento produtivo e inovativo brasileiro quanto de orientar tal desenvolvimento.

Além desse mais amplo domínio e melhor utilização do conceito, outros três desafios maiores colocam-se às políticas de promoção de arranjos e sistemas de produção e inovação no Brasil.

O primeiro diz respeito à necessidade de superar a superficialidade, miopia e imediatismo dos objetivos das políticas; reverter a destruição das capacidades locais, produtivas e inovativas; e garantir que as políticas implícitas não sejam anuladas pelas explícitas. Isto remete tanto à capacidade de desenhar e implementar políticas que sejam economicamente dinâmicas, socialmente inclusivas e politicamente viáveis, quanto à necessidade de desenhar e implementar uma política pró-ativa e de longo prazo de desenvolvimento, nas quais as demais possam se articular e sustentar. Grande parte dos insucessos das políticas públicas e privadas, especialmente aquelas para o desenvolvimento industrial e tecnológico, derivam da falta desse projeto nacional que as oriente e ancore. Qualquer política, especialmente a de promoção de arranjos e sistemas produtivos locais, será mais efetiva se representar o rebatimento - setorial, regional e local - das prioridades de um projeto de desenvolvimento nacional de longo prazo.

Em segundo lugar, coloca-se a necessidade de (i) identificar e desenhar políticas com olhar e ação sistêmi- cos, que levem em conta os requisitos dos diferentes atores locais e seus ambientes; (ii) envolver o conjunto desses atores e ambientes em seu desenho e implementação; e de (iii) garantir a coerência e a coordenação das políticas em nível local, regional, nacional e supranacional.

Em terceiro lugar coloca-se o objetivo de transformar estruturas produtivas desarticuladas e fragmentadas em sistemas dinâmicos e inovadores. Ou seja, como promover e apoiar empresas e outros atores de forma que estes se transformem em um grupo de atores que interagem e colaboram na produção, inovação, design, comercialização, etc. Um desafio associado refere-se ao objetivo de proporcionar a estes sistemas condições que lhes permitam trilhar este caminho de forma não espúria e sustentada.

De forma resumida, recomenda-se no curto prazo desenvolver os arranjos e sistemas brasileiros mobilizando sinergias, conhecimentos e capacitações para o desenvolvimento. Isto implica em apoiar os sistemas produtivos e inovativos já existentes no país, garantindo sua sustentabilidade, assim como mobilizando seus processos de aprendizado e criação de capacitações. O escopo dos casos a serem apoiados deve ser amplo e envolver atividades do setor primário, secundário e terciário. Ênfase especial deve ser dada àqueles sistemas que atendam às prioridades do desenvolvimento social e que contribuam para compensar os enormes desequilíbrios sociais e regionais brasileiros. Destaca-se, portanto, a necessidade de estimular e desenvolver tanto os arranjos e sistemas produtivos que contribuam para tais objetivos, quanto aqueles diretamente mobilizadores do desenvolvimento social e que contribuem para a melhoria das condições de vida da sociedade brasileira. Neste caso, colocam-se como carros-chefe os sistemas produtivos e inovativos nas áreas de saúde - e especialmente saúde pública - assim como os de alimentação, educação, habitação, saneamento etc.. Portanto a urgência em avançar na compreensão do desenvolvimento produtivo e inovativo destas áreas, assim como na formulação de políticas que orientem e dinamizem este desenvolvimento de forma sistêmica e sustentável.

\section{Notas}

1. Os estudos realizados no Brasil - ao mesmo tempo em que confirmam a importância da participação das universidades em diferentes sistemas produtivos e inovativos - incluem também casos onde não existem universidades, mas sim centros de formação técnica, ou mesmo nem estes, nos quais os meios de geração e difusão de conhecimentos são exclusivamente informais, mas nem por isso menos importantes ou irrelevantes para a agenda de pesquisa e de política.

2. A idéia de tecnoglobalismo é que a geração de tecnologias dar-se-ia também de forma global, com o local não apresentando importância particular.

3. Esta definição baseia-se em proposta de Lynn Mytelka (1993), suas vantagens para países menos desenvolvidos são discutidas em Cassiolato, Lastres e Maciel, 2003 e Lastres, Cassiolato e Arroio, 2005. 
4. Para detalhes sobre as convergências entre estas visões ver Cassiolato et al., 2005 e Guimarães, et al., 2006.

5. Ver www.redesist.ie.ufrj.br.

6. A descrição detalhada desta metodologia encontra-se em www.sinal.redesist.ie.ufrj.br. Ver também Lastres, Cassiolato e Campos (2006).

\section{Referências bibliográficas}

AROCENA, R.; SUTZ, J. Knowledge, innovation and learning: systems and policies in the North and in the South. In: CASSIOLATO, J.E.; LASTRES, H.M.M; MACIEL, M.M.L. (Eds.). Systems of innovation and development: evidence from Brazil. Cheltenham: Elgar, 2003.

CASSIOLATO, J.E.; LASTRES, H.M.M. Sistemas de inovação e desenvolvimento: as implicações de política. São Paulo Perspectiva, v. 19, n.1, p.34-45, Jan.-Mar. 2005.

CASSIOLATO, J.E. et al. Innovation Systems and Development: what can we learn from the Latin American experience?, III GLOBELICS CONFERENCE, Pretoria. Available at: <www.sinal.redesist ie.ufrj.br>. 2005.

CASSIOLATO, J.E.; LASTRES, H.M.M. O foco em arranjos e sistemas produtivos e inovativos locais. In: LASTRES, H.M.M.; CASSIOLATO, J.E.; MACIEL, M.L. (Eds.). Pequena empresa: cooperação e desenvolvimento local. Rio de Janeiro: Relume Dumará, 2003.

CASSIOLATO, J.E.; LASTRES, H.M.M.; MACIEL, M.M.L. (Eds.) Systems of innovation and development: evidence from Brazil. Cheltenham: Elgar, 2003.

CASSIOLATO, J.E.; LASTRES, H.M. Inovação, globalização e as novas políticas de desenvolvimento industrial e tecnológico. In: CASSIOLATO, J.E; LASTRES, H.M.M. (Eds.). Globalização e inovação localizada: experiências de sistemas locais no Mercosul, Brasília, MCT/IBICT, 1999.

COOKE, P.; MORGAN, K. The associational economy: firms, regions and innovation, New York: Oxford University Press, 1998.

DOSI, G. The nature of the innovative process. In: DOSI, G. et al. (Eds.). Technical change and economic theory. Londres: Pinter Publishers, 1988.

DOSI, G. et al. (Eds.). Technical change and economic theory. Londres: Pinter Publishers. 1988.

FAJNZYLBER, F. Competitividad internacional: evolución y leciones. Revista de la CEPAL, 36, 1988.

FREEMAN, C. Technological infrastructure and international competitiveness. Texto submetido ao OECD ad hoc group on science, technology and competitiveness. Paris: OCDE, 1982a.

FREEMAN, C. Innovation and long cycles of economic development. In: SEMINÁRIO INTERNACIONAL. Campinas: Universidade Estadual de Campinas, 1982b.

FREEMAN, C. The economics of industrial innovation. Londres: Frances Pinter, 1982c.
FREEMAN, C. Technology policy and economic performance- lessons from Japan. Londres: Frances Pinter, 1987.

FREEMAN, C. Diffusion: the spread of new technologies to firms, sectors and nations. In: HEERTJE, A. (Ed.). Innovation, Technology and Finance. Oxford, Basil Blackwell, 1988.

FREEMAN, C. The national innovation systems in historical perspective. Cambridge Journal of Economics, v.19, n.1, p.5-24, 1995.

FREEMAN, C. Innovation systems: city-state, national, continental and sub-national. In: CASSIOLATO, J.E.; LASTRES, H.M.M. (Eds.). Globalização e inovação localizada: experiências de sistemas locais no Mercosul. Brasília: MCT/IBICT, 1999.

FREEMAN, C.; PEREZ, C. Structural crises of adjustment, business cycles and investment behaviour. In: DOSI, G.; FREEMAN, C. et al. (Eds.). Technical change and economic theory. Londres: Pinter, 1988.

FREEMAN, C.; SOETE, L. The Economics of Industrial Innovation. $3^{\mathrm{a}}$ ed. Cambridge, Massachusetts: The MIT Press, 1997.

FURTADO, C. O. Capitalismo Global. São Paulo: Paz e Terra, 1998.

FURTADO, C. Desenvolvimento e subdesenvolvimento. Rio de Janeiro: Fundo de Cultura, 1961.

GEORGHIOU, L. et al. Post-innovation performance: technological development and competition. Londres: The MacMillan Press, 1986.

GUIMARÃES, V. et al. Convergências e complementaridades da corrente neo-schumpeteriana com o pensamento estruturalista de Celso Furtado. In: SABOIA, J.; CARDIM, F. (Orgs.). Celso Furtado e o Século XXI, Rio de Janeiro: Manole, 2006.

HERRERA, A. Los Determinantes Sociales de la Politica Cientifica en America Latina. In: SÁBATO, J. (Ed.). El pensamento Latinoamericano en ciencia-tecnologiadesarrollo-dependencia. Buenos Aires: Paidos, 1971.

IMAI, K-I; BABA, Y. Systemic innovation and cross-border networks. In: International Seminar on Science, Technology and Economic Growth. Paris: OCDE, 1989.

JOHNSON, B.; LUNDVALL, B.-Å. Promoting innovation systems as a response to the globalising learning economy. CASSIOLATO, J.E.; LASTRES, H.M.M.; MACIEL, M.M.L. (Eds.). Systems of innovation and development: evidence from Brazil. Cheltenham: Elgar, 2003.

JOHNSON, B. Institutional learning. In: Lundvall, B.-Å. (Ed.). National innovation systems: towards a theory of innovation and interactive learning. London: Pinter Publishers, 1992.

KLEVORICK, A.K. et al. On the sources and significance of inter-industry differences in technological opportunities. Research Policy, v.24, p.185-205, 1995.

LASTRES, H.M.M. The advanced materials revolution and the Japanese system of innovation. Londres: MacMillan, 1994. 
LASTRES, H.M.M.; CASSIOLATO, J.E; CAMPOS, R. Arranjos e sistemas produtivos e inovativos locais: vantagens do enfoque. In: LASTRES, H.M.M.E.; CASSIOLATO, J.E. (Orgs.) Estratégias para o desenvolvimento: um enfoque sobre arranjos produtivos locais do Norte, Nordeste e Centro-Oeste brasileiros. Rio de Janeiro: E-Papers, 2006.

LASTRES, H.M.M.; CASSIOLATO, J.E.; MATOS, M. Desafios do uso do enfoque em arranjos e sistemas produtivos e inovativos locais no Brasil. In: LASTRES, H.M.M.; CASSIOLATO, J.E. (Orgs.). Estratégias para o desenvolvimento: um enfoque sobre arranjos produtivos locais do Norte, Nordeste e Centro-Oeste brasileiros. Rio de Janeiro: E-Papers, 2006.

LASTRES, H.M.M., CASSIOLATO, J.E.; MACIEL, M.L. Systems of innovation for development in the knowledge era: an introduction. CASSIOLATO, J.E.; LASTRES, H.M.M.; MACIEL, M.M.L. (Eds.). Systems of Innovation and development: evidence from Brazil. Cheltenham: Elgar, 2003.

LASTRES, H.M.M.; CASSIOLATO, J.E.; ARROIO, A. Sistemas de inovação e desenvolvimento: mitos e realidades da economia do conhecimento In: LASTRES, H.M.M.; CASSIOLATO, J.E.; ARROIO, A. (Orgs.). Conhecimento, sistemas de inovação e desenvolvimento. Rio de Janeiro: UFRJ, Contraponto, 2005.

LASTRES, H.M.M.; FERRAZ, J. Economia da informação, do conhecimento e do aprendizado. In: LASTRES, H.M.M.; ALBAGLI, S. (Eds.). Informação e globalização na era do conhecimento. Rio de Janeiro: Campus, 1999.

LASTRES, H.M.M.; LEGEY, L.I.; ALBAGLI, S. Indicadores da sociedade e economia da informação, do conhecimento e do aprendizado. In: VIOTTI, E.; MACEDO, M. Indicadores de ciência, tecnologia e inovação. Campinas: Unicamp, 2003.

LUNDVALL, B.- $\AA$. Product innovation and userproducer interaction. Aalborg: Aalborg University Press, 1985.

LUNDVALL, B.-A. Innovation as an interactive process: from user-producer interaction to the national innovation systems. In: DOSI, G. et al. (Eds.). Technical change and economic theory. Londres: Pinter Publishers, 1988.

LUNDVALL, B.-Å. Introduction. In: Lundvall, B.-Å. (Ed.). National systems of innovation: towards a theory of innovation and interactive learning. Londres: Pinter, 1992.

LUNDVALL, B.- $\AA$. The social dimension of the learning economy. DRUID, Working Paper 96-1, Aalborg University, Aalborg, 1995.

LUNDVALL, B.-A. National innovation system: analytical policy device and policy learning tool. Mimeo. Department of Business Studies, Aalborg University, Aalborg, Denmark, 2006.

MALERBA, F; ORSENIGO, L. The dynamics and evolution of industries. Industrial Corporate Change, v.5, n. 1, p.51-87, 1996.

MARQUES, I. Desmaterialização e trabalho. In: LASTRES, H.M.M; ALBAGLI, S. (Eds.). Informação e globalização na era do conhecimento. Rio de Janeiro: Campus, 1999.

MARSHALL, A. Industry and trade: a study of industrial technique and business organization, and of their influence on the conditions of various classes and nations. Londres: MacMillan, 1919.

METCALFE, J. Technological innovation and the competitive process. In: Hall, P. (Ed.). Technology, innovation and economic growth. Southampton: Camelot Press, 1986.

MOWERY, D.; OXLEY, J. Inward technology transfer and competitiveness: the role of national systems of innovation. Cambridge Journal of Economics, v.19, n.1, p.67-93, 1995.

MYTELKA, L. A role for innovation networking in the other two-thirds. Futures, Jul.-Ago, 1993.

MYTELKA, L.K. Local systems of innovation in a globalized world economy. Industry and Innovation, v.7, n.1, p.15-32, 2000

NELSON, R. (Ed.). National innovation systems: a comparative analysis. Oxford: Oxford University Press, 1993.

OCDE. Technical change and economic policy. Paris: OCDE, 1980 .

OCDE. New technologies in the 1990s: a socio-economic strategy (Sundqvist Report). Paris: OCDE, 1988.

OCDE. Proposed guidelines for collecting and interpreting innovation data (Oslo Manual). Paris: OCDE, 1992a

OCDE. Technology and the economy: the key relationships. Paris: OCDE, 1992b.

OCDE. Technological innovation: some definitions and building blocks. Draft Background Report, Chapter 2, Technology/Economy Programme. Paris: OCDE, 1990.

PEREZ, C. New technologies and development. In: FREEMAN, C.; LUNDVALL, B.-A. (Eds.). Small countries facing the technological revolution. Londres: Pinter Publishers, 1988.

PEREZ, C. Structural change and the assimilation of new technologies in the economic and social systems. Futures, v. 15, n.5, 1983.

POLANYI, M. The tacit dimension. Londres: Routledge; Kegan, 1966.

PREBISCH, R. O desenvolvimento econômico da América Latina e alguns de seus problemas principais. In: BIELSCHOWSKY, R. (Org.) Cinqüenta anos de pensamento na CEPAL. Record: Rio de Janeiro [2000] 1949.

REINERT, E; REINERT, S. GLOBELICS CONFERENCE, 1, 2003, Rio de Janeiro.

ROSENBERG, N. Perspectives on technology. Cambridge: Cambridge University Press, 1976.

ROTHWELL, R. et al. Updated - project SAPPHO phase II. Research Policy, v.3. 1974. p.258-291.

SCHMITZ, H.; CASSIOLATO, J.E. Hi-tech for industrial development lessons from the Brazilian 
experience in electronics and automation. Londres: Routledge, 1992.

SCHUMPETER, J.A. The theory of economic development. Cambridge: Harvard University Press, 1912.

SCHUMPETER, J.A. Business cycles: a theoretical, historical and statistical analysis of the capitalist process, 2v. New York: McGraw-Hill, 1939.
SCHUMPETER, J.A. Capitalism, socialism and democracy. New York: Harper, 1942.

TEECE, D. Technological change and the nature of the firm. In: DOSI, G. et al. (Eds.). Technical change and economic theory. Londres: Pinter Publishers, 1988.

\section{Sobre os autores}

\section{José Eduardo Cassiolato}

Doutor em Desenvolvimento, Industrialização e Política Científica e Tecnológica e Mestre em Economia do Desenvolvimento pela Sussex University, Inglaterra e Economista pela Universidade de São Paulo (USP). Atualmente é professor e pesquisador do Instituto de Economia (IE/UFRJ) onde coordena a RedeSist e um projeto de pesquisa internacional sobre sistemas de inovação no Brasil, Rússia, Índia, China e África do Sul. Além disso, é membro do Conselho Científico da rede mundial Globelics, Professor Convidado do Mestrado Profissional em Gestão de C\&T em Saúde da Escola Nacional de Saúde Pública da Fundação Oswaldo Cruz; e da Escola de Pós-graduação em Inovação e Desenvolvimento Econômico: Globelics Academy de Lisboa em Portugal. Dedica-se à pesquisa e ensino em economia da inovação, do desenvolvimento e política industrial e de C\&T\&l.

\section{Helena Maria Martins Lastres}

Doutora em Desenvolvimento, Industrialização e Política Científica e Tecnológica da Sussex University na Inglaterra; Mestre em Engenharia da Produção pela COPPE/UFRJ e Economista, Faculdade de Economia, Administração/UFRJ. Atualmente é professora, pesquisadora e coordenadora da RedeSist, UFRJ. Além disso é pesquisadora Titular do Instituto Brasileiro de Informação Científica e Tecnológica do MCT e professora convidada do Mestrado Profissional em Gestão de C\&T em Saúde, Escola Nacional de Saúde Pública da Fundação Oswaldo Cruz; e da Escola de Pós-graduação em Inovação e Desenvolvimento Econômico: Globelics Academy de Lisboa em Portugal. Dedica-se à pesquisa e ao ensino em política de desenvolvimento industrial e tecnológico; economia da inovação, da informação e do conhecimento e arranjos e sistemas produtivos e inovativos locais. 